\title{
Achtsamkeit in Gruppensituationen und Beratungsprozessen
}

\author{
Telse A. Iwers-Stelljes
}

Online publiziert: 20. August 2014

(C) Springer Fachmedien Wiesbaden 2014

Achtsamkeit ist als basale Haltung der Selbstwahrnehmung und Reflexion wie auch der Interaktionswahrnehmung und -gestaltung allgemein anerkannt. Die aktuelle Diskussion legt es allerdings nahe, an der Entwicklung eindeutiger Verständigungsgrundlagen zu arbeiten. Dazu bedarf es theoretischer Auseinandersetzungen und Diskurse, um Trennlinien und Abgrenzungen zu alltagsweltlich genutzten und wenig abgesicherten Verfahren herauszuarbeiten und fundierte Begriffsklärungen unter Berücksichtigung historischer Entwicklungslinien und aktueller Konzepte $\mathrm{zu}$ fokussieren. Ebenso sind Untersuchungen zur Wirksamkeit von wissenschaftlich begründet entwickelten Achtsamkeitstrainings notwendig, die ihrerseits Kriterien wissenschaftlicher Evaluationen standhalten und dazu beitragen, Verfahren der Achtsamkeitssteigerung als begründete Methoden der Entwicklung von Selbst- und Sozialkompetenz auszuweisen und diese in pädagogischen Handlungskontexten zu lozieren. Insgesamt soll die wissenschaftliche Präzisierung vorangebracht und Achtsamkeit als Konzept der Evaluation und Intervention pädagogischer Professionalisierung fruchtbar gemacht werden. Dazu möchte der Thementeil dieses Heftes beitragen, und zwar unter besonderer Berücksichtigung der Auswirkungen von Achtsamkeitskonzepten auf die Gestaltung von pädagogisch-psychologisch begründeten Interaktionssituationen.

Die Beiträge, welche hier zusammengetragen werden, verfolgen dem entsprechend zwei Richtungen: Zum einen werden Definitionen aus verschiedenen Perspektiven beleuchtet und Konzeptualisierungen präsentiert, um den theoretischen Diskurs $\mathrm{zu}$ fördern und empirisch zu verdichten. Zum anderen werden spezifische Handlungsfelder und Zielgruppen in den Blick genommen, in denen Achtsamkeitsprinzi-

Priv.Doz. Dr.phil.habil. T. A. Iwers-Stelljes ( $\square$ )

Universität Hamburg,

Hamburg, Deutschland

E-Mail: Telse.Iwers-Stelljes@uni-hamburg.de 
pien und deren Förderung auf ihre jeweilige Wirksamkeit hin zunächst intrasubjektiv und dann auch intersubjektiv als Sozialkompetenzentwicklung im Hinblick auf die professionelle Haltung in Interaktionen mit Individuen und Gruppen reflektiert und diskutiert werden. Insgesamt wird eine Verschränkung theoretischer, praktischer und empirischer Zugänge vorgelegt, welche die Achtsamkeit als wesentliches Professionalisierungskonzept von PädagogInnen ausweisen soll.

\section{Texte}

Telse Iwers-Stelljes \& Carolin Pollok zeigen in ihrem Beitrag Lösung von mentalen Konflikten und Steigerung von Achtsamkeit in Entscheidungsprozessen am Beispiel von Entscheidungssituationen auf, wie hochgradig konfliktbehaftet Prozesse der Entscheidungsfindung und Handlungsregulation sein können, um daran anschließend die spannungsreduzierende Wirksamkeit eines wissenschaftlich begründeten Verfahrens der Selbstregulation zur Entwicklung situationsangemessener Achtsamkeitsentwicklung zu skizzieren, welches u. a. in Beratungskontexten erprobt wurde.

In einer theoretischen und empirischen Untersuchung berichten Janike Tammena \& Telse Iwers-Stelljes anschließend unter dem Titel Achtsamkeit in der Beratung zunächst von Parallelen zwischen Achtsamkeit im Buddhismus, in westlichen Philosophien und in der psychologischen Forschung. Es wird eine Zusammenfassung grundlegender traditions- und theorieübergreifender Achtsamkeitsdimensionen herausgearbeitet. Das so entwickelte Achtsamkeitsmodell wird in einer qualitativen Studie empirisch überprüft und zeigt zugleich die immanente Nutzung von Achtsamkeitskriterien auch in nicht explizit als solche bezeichneten Beratungskonzepten.

Das Alter, d. h. allgemein die nachberufliche Lebenszeit, gewinnt in unserer Gesellschaft zunehmend an Bedeutung, denn diese Lebensphase nimmt inzwischen oftmals fast ein Drittel der Erwachsenenlebenszeit ein. Bislang eher kriseninterventiv angelegte Beratungskonzepte werden von Hartmut Frech in seinem Beitrag Achtsamkeit in der Beratung von Älteren erweitert um einen durch Achtsamkeit geprägten Beratungsansatz, der sich grundlegenden Fragen und Herausforderungen des Alter(n)s widmet.

Adriane Kobusch plädiert in ihrem Beitrag Achtsamkeit in der Schule durch ein professionsbezogenes Achtsamkeitstraining für LehrerInnen zunächst für den Transfer von Achtsamkeitsprinzipien und die Entwicklung einer ,Achtsamkeitskultur' in den schulischen Kontext und fokussiert auf das Üben einer achtsamen Haltung und deren Auswirkungen. Dabei stellt sie die zentrale Funktion der LehrerInnen heraus und berichtet von einem Forschungsprojekt der Universität Bielefeld zur Achtsamkeitsentwicklung von LehrerInnen.

Alexander Gröschner, Tina Seidel, Ann-Kathrin Pehmer \& Katharina Kiemer nehmen eine ergänzende Perspektive ein und berichten unter dem Titel Facilitating collaborative teacher learning: The role of ,mindfulness “ in video-based teacher professional development programs (Förderung kollaborativen Lernens von Lehrpersonen: Zur Rolle von ,Achtsamkeit“ in videobasierten Fortbildungsprogrammen) von einem Verfahren der achtsamen Moderation in der Fortbildung von Lehrern. Das Fortbildungsprogramm „Dialogic Video Cycle“ (DVC) zielt auf die Verbesserung 
des dialogischen Unterrichtsgesprächs ab. Die Autorinnen zeigen die Bedeutsamkeit eines achtsamen Moderators im Hinblick auf den Erwerb neuen Wissens und die Förderung des Austausches in der Fortbildungsgruppe.

Insgesamt verweisen die Beiträge auf Forschungsaktivitäten im Bereich grundlegender Konzeptualisierungen ebenso wie im Bereich der praktischen Bedeutsamkeit und Förderung von Achtsamkeit. 